\title{
Parto humanizado y violencia obstétrica en Chile: Percepción de los actores involucrados
}

\author{
Humanized childbirth and obstetric violence in Chile: Stakeholders' perceptions
}

\section{Comentado de:}

Silva $A$ et al. Medwave 2020; 20(9):e8047. PMID: 33141815. DOI: $10.5867 /$ medwave.2020.09.8047 1

\section{Objetivo}

Conocer y analizar las percepciones de los actores involucrados en la asistencia sanitaria y la gestión del parto acerca de la violencia obstétrica y el parto humanizado.

\section{Diseño}

Revisión panorámica.

\section{Fuentes de datos}

La revisión incluyó dos tipos de fuentes:

1. Análisis de estudios primarios y secundarios desarrollados en Chile: artículos científicos, informes de investigación y textos de análisis realizados en Chile, extraídos de bases de datos como: BEIC, BIREME, Cochrane Library, Dialnet, EBSCO, Web of Science, MEDLINE/PubMed y Redalyc, con los que se buscó reflejar el punto de vista de la comunidad científica acerca del parto respetado/humanizado y la violencia obstétrica. La búsqueda inicial fue seguida por la técnica de búsqueda en cadenas o bola de nieve.

2. Análisis de declaraciones de otros actores, para reflejar su punto de vista: textos, declaraciones, informes y proyectos de ley publicados en sitios web de instituciones gubernamentales, organizaciones sociales, asociaciones profesionales y actores políticos.

\section{Selección de estudios}

Los estudios que reflejaban el punto de vista de la comunidad científica fueron seleccionados según los siguientes criterios de elegibilidad:

- Tema: fueron considerados elegibles aquellos que abordaban las dimensiones del parto humanizado, respetado o personalizado, la violencia obstétrica y el maltrato en la atención del parto, y los que abordaron otros tópicos en la medida en que contribuyeron con el objetivo del estudio, como, p. ej., el desarrollo histórico y cultural de la matronería profesional en Chile.

- Tipo de trabajo y formato de publicación: fueron elegibles todos los trabajos científicos y documentos de análisis, sin restricción disciplinaria: estudios primarios de diverso diseño (cuantitativos, cualitativos, mixtos), estudios secundarios, revisiones (narrativa, sistemática, meta-análisis), publicados en revistas científicas, informes de investigación, libros y capítulos de libros.

- Idioma: fueron elegibles textos en español, inglés, portugués, francés y alemán desarrollados en Chile entre los años 2000 y 2019.

- Actores: fueron incluidos los textos que reflejaban el punto de vista gubernamental, de la sociedad civil y las asociaciones de profesionales.

Fueron excluidos los artículos cuya temática abordara el evento de aborto (aun cuando el concepto de violencia obstétrica incluye la acción durante el aborto) por exceder el foco de la esta investigación; las tesis y presentaciones a congresos, con la excepción de un estudio antropológico de trascendencia nacional sobre el parto humanizado y la violencia obstétrica, y los textos de los medios de comunicación masiva y las redes sociales, con excepción de la red social de la Red Latinoamericana y del Caribe para la Humanización del Parto y el Nacimiento (RELACAHUPAN).

\section{Análisis de datos}

El análisis del conjunto de la información se realizó mediante estrategias para contenido cualitativo, inductivo, temático. Inicialmente, los documentos fueron analizados para identificar características descriptivas, temas y subtemas. Luego, del análisis integrado de los textos emergió un conjunto de categorías de análisis explicativas. La codificación fue realizada y discutida por cuatro autores: dos para los estudios cualitativos y dos para los estudios cuantitativos. La codificación de las declaraciones de los actores fue realizada por tres autores de distinto perfil profesional.

La extensión PRISMA para revisiones panorámicas fue utilizada para delinear los estándares de esta revisión panorámica. La calidad de los estudios incluidos no fue evaluada.

\section{Resultados Principales}

En total fueron incluidos 70 documentos publicados entre 2000 y 2019; 38 documentos publicados por la comunidad científica y 32 documentos generados por los actores involucrados.

El estudio integrado de los documentos de la comunidad científica reveló tres categorías de análisis centrales abordadas por los investigadores: parto humanizado, satisfacción de la usuaria, y construcción histórico-cultural del modelo biomédico predominante de atención del parto. De estas categorías surgió que, pese a la existencia de un Manual de atención personalizada en el proceso reproductivo publicado en 2008 por el Ministerio de Salud de Chile, continúa siendo elevada la tasa de cesáreas, con escasa incorporación de las prácticas recomendadas y con frecuente insatisfacción de las usuarias en relación al trato recibido.

El análisis de los actores evidenció que, desde el punto de vista gubernamental, el Ministerio de Salud ha abordado el tema de la violencia obstétrica desde la elaboración de Guías, pero el Instituto Nacional de Derechos Humanos ha destacado la persistencia de condiciones de trabajo desfavorables para el personal de salud, la violencia de género proveniente del contexto cultural nacional y la lentitud en la implementación de las recomendaciones ministeriales sobre el parto personalizado.

En cuanto al punto de vista de los profesionales de la salud sobre la violencia obstétrica, los autores describen las declaraciones de dos organizaciones, quienes en conjunto tienden o bien a rechazar el término violencia obstétrica, a minimizarlo, o a ligarlo a prácticas o condiciones ajenas a los profesionales representados por la sociedad en cuestión (tales como las condiciones laborales), así como a rechazar la promulgación de leyes que puedan regular este aspecto de la actividad profesional.

Luego del análisis, los autores generaron una tabla resumiendo las áreas en las cuales habría mayor probabilidad de lograr consensos, así como aspectos que darían lugar a discusión entre los distintos actores (Ver Tabla 1). 
Tabla 1. Áreas de consenso y disidencia sobre el parto humanizado y la violencia obstétrica en Chile

\begin{tabular}{|l|l|l|}
\hline Tópicos & $\begin{array}{l}\text { Consenso en materias que han de ser abordadas e } \\
\text { implementadas }\end{array}$ & $\begin{array}{l}\text { Ámbitos con probabilidad de generar disenso y discu- } \\
\text { sión }\end{array}$ \\
\hline Metas de la atención del parto & $\begin{array}{l}\text { Calidad de la atención del parto. Bienestar maternal, } \\
\text { satisfacción de la usuaria. }\end{array}$ & $\begin{array}{l}\text { Cambio de paradigma en el modelo de atención (desde } \\
\text { tecnocrático a personalizado). }\end{array}$ \\
\hline Políticas públicas de salud & $\begin{array}{l}\text { Política pública para implementar las orientaciones de } \\
\text { parto personalizado. Adaptación de la infraestructura y } \\
\text { el equipamiento hospitalario. Adecuación de recursos } \\
\text { humanos y manejo del estrés laboral. Plan de parto } \\
\text { desde el primer nivel de atención. }\end{array}$ & $\begin{array}{l}\text { Cambio en la estructura público-privada de organización } \\
\text { del trabajo médico, y reducción de inequidades en aten- } \\
\text { ción de salud. Medidas de desincentivo de realización } \\
\text { de cesáreas. Ley para asegurar la atención personali- } \\
\text { zada del parto y enfrentar la violencia obstétrica. }\end{array}$ \\
\hline Exigencias académicas & $\begin{array}{l}\text { Innovación en currículo académico de las escuelas de } \\
\text { medicina y obstetricia (matronería). Investigación en } \\
\text { los tipo de parto y los modelos de atención. }\end{array}$ & $\begin{array}{l}\text { Formación para el desarrollo de competencias de tra- } \\
\text { bajo en equipo entre médicos obstetras y matronas, y } \\
\text { desarrollo de una matronería más autónoma. Inclusión } \\
\text { de la dimensión de género en la formación universitaria. }\end{array}$ \\
\hline Cambio cultural & $\begin{array}{l}\text { Información y educación de la mujer, la pareja y la } \\
\text { familia en la materia. Cambio de actitud del equipo de } \\
\text { salud hacia el buen trato de las madres durante el pro- } \\
\text { ceso de parto. }\end{array}$ & $\begin{array}{l}\text { Inclusión de la dimensión de género en la atención del } \\
\text { parto y en la relación del profesional de la salud con la } \\
\text { mujer. }\end{array}$ \\
\hline
\end{tabular}

\section{Conclusiones}

El análisis de la perspectiva de los actores permitió identificar áreas de conflicto y de consenso, así como diversas dimensiones interactuantes que obstaculizan el avance hacia la humanización de la atención del parto. Esta estrategia de análisis contribuye a la identificación de aspectos críticos a ser abordados para un desarrollo de políticas sanitarias integrales y efectivas.

Fuente de financiamiento / Conflicto de interés de los autores : Este estudio fue apoyado por la Comisión Nacional de Investigación Científica y Tecnológica (CONICYT), Ministerio de Educación de Chile, Fondo Nacional de Investigación en Salud FONIS. Los autores no declaran conflictos de interés.

\section{Comentario}

Esta revisión panorámica permite visualizar el debate social existente acerca del parto respetado y la violencia obstétrica, y la lucha impulsada por las diversas organizaciones de la sociedad civil que pretenden transformar las prácticas opresoras sobre los cuerpos de las personas gestantes durante sus procesos de embarazo, trabajo de parto, parto y puerperio, en prácticas garantes de derechos. Hasta la fecha, Chile no cuenta con una legislación orientada específicamente a la protección de los derechos de estas personas, y la ley $\mathrm{N}^{\circ} \mathbf{2 0 . 5 8 4}$ de Derechos y Deberes de las personas en relación con acciones vinculadas a su atención de Salud ${ }^{2}$ resulta insuficiente para el abordaje de esta problemática. En la actualidad se está llevando a cabo en el Congreso el debate de un proyecto de ley elaborado en el año 2018, denominado Ley Adriana, que persigue lograr la garantía de derechos en esta materia, si bien aún no se han logrado suficientes avances para su aprobación ${ }^{3,4}$.

Al analizar la situación de Argentina, el país cuenta con dos leyes en torno a esta temática. Por un lado, la ley №25.929 de parto humanizado, que lo define como la atención del parto respetuoso de los deseos, los tiempos biológicos-psicológicos y las necesidades de la persona gestante y su familia, garante de la toma de decisiones seguras e informadas y dirigido a evitar todo tipo de intervenciones innecesarias ${ }^{5}$. Por otro lado, la ley $\mathrm{N}^{\circ} 26.485$ de Protección integral a la mujeres, la cual en su inciso 6 sanciona la violencia obstétrica, y la define como aquella ejercida por el personal de salud sobre el cuerpo y los procesos reproductivos de las mujeres, expresada en un trato deshumanizado, un abuso de medicalización y patologización de los procesos naturales ${ }^{6}$. Sin embargo, pese a esta legislación vigente, los indicadores del Sistema de Información Perinatal permiten visibilizar la magnitud de los desafíos pendientes. Por ej., en la provincia de Buenos Aires persisten aún prácticas contrarias al parto respetado tales como una elevada tasa de cesáreas (que en el sector público pasó de $27 \%$ en 2011 a $36 \%$ en 2019) y la falta de acompañamiento en el parto (44\% en 2019$)^{7}$.

Respecto a la metodología empleada en esta revisión, cabe destacar que no fueron evaluados la calidad de la evidencia y el riesgo de sesgo de cada artículo revisado, ya que los resultados no estaban destinados a resolver preguntas o establecer recomendaciones clínicas, sino a presentar una visión general de la evidencia en la materia ${ }^{8}$.

Una de las principales limitaciones de la revisión resumida fue la falta de inclusión de documentos provenientes del sector privado de salud, de los medios de comunicación y de fuentes adicionales (como p.ej., testimonios en redes sociales o centro de reclamos/sugerencias de instituciones hospitalarias). Sin embargo, es importante destacar que permitió una exploración extensa de las percepciones existentes, aportando a la compresión de la problemática con el fin de pensar estrategias para el desarrollo de políticas públicas de salud.

\section{Conclusiones del comentador}

Esta revisión panorámica exploró las percepciones de los diversos actores involucrados en el parto respetado y la violencia obstétrica en Chile. Tras el análisis de los documentos incluidos, se identifica la necesidad de contar tanto con la consolidación de un marco legal capaz de regular y sancionar las diferentes prácticas en esta materia, como con el compromiso de las diversas instituciones de salud, organizaciones gubernamentales y asociaciones civiles para lograr cambiar aquellas 
prácticas que producen y reproducen la violencia obstétrica.

La violencia obstétrica no es solo una modalidad de la violencia de género, sino también un problema de salud pública. Persiste el desafío de consolidar un proceso de transformación de las prácticas patologizantes, medicalizantes y de sobreintervención del embarazo y el parto, reivindicando el rol protagónico de las personas gestantes y adecuando estas experiencias a sus deseos, necesidades, decisiones y singularidades, en el marco de un trato digno, amable y respetuoso, en todos los ámbitos donde se efectúe su atención.

Eros Miguel Angeletti [ Servicio de Medicina Familiar y Comunitaria, Hospital Italiano de Buenos Aires. eros.angeletti@hospitalitaliano.org.ar ]

Angeletti E Parto humanizado y violencia obstétrica en Chile: Percepción de los actores involucrados. Evid Actual Pract Ambul. 2022;25(1):e002153. Available from: https://dx.doi.org/10.51987/EVIDENCIA.V25I1.6998. Comentado de: Silva A et al. Percepción de actores involucrados acerca del parto humanizado y la violencia obstétrica en Chile: una revisión panorámica. Medwave 2020; 20(9):e8047. PMID: 33141815. DOI: 10.5867/medwave.2020.09.8047

\section{Referencias}

1. Silva A, Pantoja F, Millón Y, et al. Percepción de actores involucrados acerca del parto humanizado y la violencia obstétrica en Chile: una revisión panorámica. Medwave. 2020;20(9):e8047. Available from: 10.5867/medwave.2020.09.8047.

2. Ministerio del Interior y Seguridad Pública Diario Oficial de la República de Chile . Ley $N^{\circ}$ 20584. Derechos y deberes que tienen las personas en relación con acciones vinculadas a su atención en salud.; 2012.

3. Congreso Nacional de Chile Plataforma de Participación ciudadana del Congreso Nacional de Chile . Ley acerca de la violencia gineco obstétrica y el incentivo al parto respetado. ; 2021. Available from: https://congresovirtual.cl/project/3825.

4. Congreso Nacional de Chile Cámara de Diputados. Proyecto de Ley parto respetado y fin a la violencia obstétrica. Boletín N $12148-11 ; 2018$. Available from: https://www.camara.cl/legislacion/ProyectosDeLey/tramitacion.aspx?prmID=12670\&prmBoletin=12148-11.

5. Argentina. Ministerio de Justicia y Derechos Humanos de la Nación. Ley $N^{\circ}$ 25.929: Ley de parto humanizado.; 2004. Available from: http://servicios. infoleg.gob.ar/infolegInternet/anexos/95000-99999/98805/norma.htm.

6. Argentina. Ministerio de Justicia y Derechos Humanos de la Nación. Ley N ${ }^{\circ}$ 26.485: Ley de protección integral para prevenir, sancionar y erradicar la violencia contra las mujeres en los ámbitos en que desarrollen sus relaciones interpersonales; 2009. Available from: http://servicios.infoleg.gob. ar/infoleglnternet/anexos/150000-154999/152155/norma.htm.

7. Provincia de Buenos Aires. Ministerio de Salud. Guía de implementación del parto respetado en la Provincia de Buenos Aires en el marco de la Ley Nacional $\mathrm{N}^{\circ} 25.929 ; 2021$. Available from: https://ministeriodelasmujeres.gba.gob.ar/gestor/uploads/guiapartorespetado.pdf.

8. Verdejo C, Tapia-Benavente L, Schuller-Martínez B, et al. Lo que tienes que saber sobre las revisiones panorámicas. Medwave. 2021;21(2):e8144 Available from: 10.5867/medwave.2021.02.8144. 